First publ. in: Physical Review B, Vol. 72 (2005), Article 134522

\title{
Full counting statistics of Andreev scattering in an asymmetric chaotic cavity
}

\author{
Mihajlo Vanević and Wolfgang Belzig \\ Departement für Physik und Astronomie, Klingelbergstrasse 82, 4056 Basel, Switzerland \\ (Received 13 December 2004; revised manuscript received 18 July 2005; published 25 October 2005)
}

\begin{abstract}
We study the charge transport statistics in coherent two-terminal double junctions within the framework of the circuit theory of mesoscopic transport. We obtain the general solution of the circuit-theory matrix equations for the Green's function of a chaotic cavity between arbitrary contacts. As an example we discuss the full counting statistics and the first three cumulants for an open asymmetric cavity between a superconductor and a normal-metal lead at temperatures and voltages below the superconducting gap. The third cumulant shows a characteristic sign change as a function of the asymmetry of the two quantum point contacts, which is related to the properties of the Andreev reflection eigenvalue distribution.
\end{abstract}

PACS number(s): 74.50.+r, 72.70.+m, 73.23. $-\mathrm{b}, 05.40 .-\mathrm{a}$

\section{INTRODUCTION}

Mesoscopic heterojunctions with superconductor (S) and normal-metal or semiconductor $(\mathrm{N})$ leads exhibit a rich variety of phenomena that have been studied through conductance and noise for some time already; ${ }^{1-13}$ see also Refs. 14-18. In the absence of disorder charge transport through an interface between $\mathrm{S}$ and $\mathrm{N}$ can be described by the Blonder-Tinkham-Klapwijk (BTK) model, ${ }^{1}$ while the effects of scattering by impurities and interface roughness can be taken into account using the scattering theory of mesoscopic transport. ${ }^{5-8,14-17}$ Recent experimental advancements ${ }^{19-27}$ raised interest in the statistical properties of the charge transfer in mesoscopic systems. Current noise contains information on the temporal correlation of quasiparticles relevant for transport and originates from the fluctuations of quasiparticle occupation numbers or random scattering at barriers or impurities. Further, it can be used to probe internal energy scales of the system or the effective charge of the elementary transport mechanisms, ${ }^{15-18}$ as well as to detect the correlations intrinsic to the many-body state of entangled systems. ${ }^{18}$

If the charge transfer events are uncorrelated, the zerofrequency noise power at low temperatures (shot noise) has the Poisson value $P_{I}=2 e I$, where $e$ is electron charge and $I$ is the average current through the sample. This is the case for the charge transport in a low-transparency normal-state tunnel junction at zero temperature and low bias. In less-opaque normal-state junctions shot noise is usually suppressed below the Poisson value, with the deviations characterized by the Fano factor $F=P_{I} / 2 e I=\left[\Sigma_{n} T_{n}\left(1-T_{n}\right)\right] / \Sigma_{n} T_{n}$, where $T_{n}$ are transmission eigenvalues of the junction. For a metallic diffusive wire ${ }^{19,20}$ and for an open symmetric chaotic cavity ${ }^{21}$ Fano factors have universal values ${ }^{16,28-30} F=1 / 3$ and $F$ $=1 / 4$, respectively, which do not depend on microscopic properties like the impurity concentration or geometry of the sample.

The effect of superconductivity leads to a doubled shot noise in subgap transport through low-transparency S/N tunnel junctions ${ }^{24}$ and in diffusive normal wires in contact with a superconductor. ${ }^{22,23}$ This doubling can be understood as a consequence of the effective charge doubling in the Andreev process. ${ }^{31}$ In general, however, the noise is also affected by the change of transmission properties of the structure due to the superconductor proximity effect and the doubling is not generic. For example, in an open symmetric cavity with a superconducting and normal-metal lead the Fano factor has the value ${ }^{15} F \approx 0.60$ which is more than 2 times larger than in the corresponding normal-state junction $(F=1 / 4)$, in agreement with recent experimental results. ${ }^{25}$ At bias voltages on the order of the superconducting gap both normal and Andreev scattering processes contribute to transport ${ }^{32,33}$ and the picture of the effective charge carriers fails. ${ }^{34}$

The statistical theory of mesoscopic transport, ${ }^{35}$ full counting statistics, provides the complete characterization of the charge transfer and has led to a new and fundamental understanding of quantum transport phenomena in nanoscale conductors. ${ }^{33,36}$ Higher-order moments of the charge transport statistics may provide additional information to the current noise measurements. ${ }^{35,37,38}$ The third-order correlations of voltage fluctuations across the nonsuperconducting tunnel junctions have been measured by Reulet et al. ${ }^{26}$ and recently by Bomze et al. ${ }^{27}$ the latter confirming the Poisson statistics of the charge transfer at negligible coupling of the system to environment. The semiclassical theory of higher-order cumulants based on the Boltzmann-Langevin equations has been developed recently by Nagaev et al. ${ }^{39}$ The quantummechanical approach to full counting statistics based on the extended Keldysh-Green's function technique ${ }^{40,41}$ in the discretized form of the circuit theory ${ }^{42,43}$ was put forward for multiterminal circuits by Nazarov and Bagrets. ${ }^{44}$ This approach can describe junctions with different connectors and leads as well as multiterminal circuits in unified and very general way. Within the circuit theory, the doubling of shot noise (i.e., $F=2 / 3$ ) was found in diffusive $\mathrm{S} / \mathrm{N}$ junctions in both the fully coherent $e V \ll E_{T h}$ and the completely incoherent $e V \gg E_{T h}$ regimes, ${ }^{45,46}$ where $E_{T h}$ is the inverse diffusion time. At intermediate bias voltages $e V \sim E_{T h}$, the transport is affected by electron-hole coherence leading to an enhancement of the differential Fano factor. ${ }^{46,47}$ The influence of coherence effects on the full counting statistics and noise in other single and double $\mathrm{S} / \mathrm{N}$ junctions was studied in Refs. 48-50. The current correlations in a three-terminal chaotic cavity operated as superconducting beam splitter were studied by Samuelsson and Büttiker, ${ }^{51}$ yielding unusual positive 


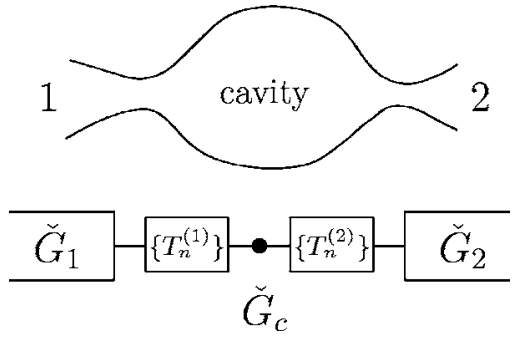

FIG. 1. A chaotic cavity coupled to the leads by two junctions with transmission eigenvalues $\left\{T_{n}^{(1)}\right\}$ and $\left\{T_{n}^{(2)}\right\}$ (top). The discrete circuit-theory representation of the system is shown in the lower part. The leads and cavity are characterized by the corresponding matrix Green's functions. The junctions, depicted as connectors, carry conserved matrix currents.

cross correlations. From a study of the full counting statistics these positive correlations can be attributed to the uncorrelated injection of Cooper pairs. ${ }^{52}$

In this article we study the full counting statistics of coherent charge transport in a chaotic cavity using the circuit theory of mesoscopic transport. We show that the system of matrix equations for the Green's function of the cavity can be solved, effectively, as a system of scalar equations independently of the type of leads and without resorting to the matrix components or parameterizations. As an application we find the Green's function for an open asymmetric cavity between arbitrary leads. For the special case of a cavity between the superconductor and normal metal, we find the cumulant generating function and the first three cumulants and discuss the interplay between superconducting proximity effect and scattering properties of the junction. The results are compared with those for a normal-state junction ${ }^{38}$ and for different couplings of a cavity to the leads. Current correlations in a structure with high-quality contacts between a cavity and superconductor have been studied experimentally by Choi et al. ${ }^{25}$ recently.

\section{MODEL}

The system we consider is a chaotic cavity coupled to two leads by mesoscopic junctions characterized by the transmission eigenvalues $\left\{T_{n}^{(1)}\right\}$ and $\left\{T_{n}^{(2)}\right\}$, respectively. Charging of the cavity is negligible if the cavity is large enough and the conductances of the junctions $g_{1,2}$ are much larger than the conductance quantum $g_{0}=2 e^{2} / h$. We assume an isotropic quasiparticle distribution function inside the cavity due to chaotic scattering. The decoherence effects as well as the energy dependence of transmission eigenvalues can be neglected if the total dwell time in the cavity is small with respect to time scales set by the inverse temperature and bias voltage. We apply the circuit theory of mesoscopic transport ${ }^{43}$ with the specific parts of the system represented by the corresponding discrete circuit elements, as shown in Fig. 1. The leads are characterized by known quasiclassical matrix Green's functions $\check{G}_{1,2}$ which depend on the quasiparticle energy, lead temperature, chemical potential, and counting field and satisfy the normalization condition $\check{G}_{1}^{2}=\check{G}_{2}^{2}=1$.
The lead Green's functions are not necessarily the ones of bulk electrodes-for example, they can be nodes that are part of a larger circuit. In the following we will refer to these nodes as leads. The formulation below is independent of the concrete matrix structure, provided the "lead" Green's functions obey the normalization conditions. The chaotic cavity is represented as an internal node associated with an unknown Green's function $\breve{G}_{c}$, which will be obtained from the matrix current conservation and the normalization condition $\check{G}_{c}^{2}=1$. Left and right junctions $(i=1,2)$, depicted as connectors, carry matrix currents ${ }^{43}$

$$
\check{I}_{i}=\frac{2 e^{2}}{h} \sum_{n} \frac{2 T_{n}^{(i)}\left[\check{G}_{i}, \check{G}_{c}\right]}{4+T_{n}^{(i)}\left(\left\{\check{G}_{i}, \check{G}_{c}\right\}-2\right)},
$$

which flow from the cavity into the leads. The current conservation $\check{I}_{1}+\check{I}_{2}=0$ for the Green's function $\check{G}_{c}$ of the cavity reduces to

$$
\left[\check{p}_{1} \check{G}_{1}+\check{p}_{2} \check{G}_{2}, \check{G}_{c}\right]=0
$$

with

$$
\check{p}_{i}=\sum_{n} \frac{T_{n}^{(i)}}{4+T_{n}^{(i)}\left(\left\{\check{G}_{i}, \check{G}_{c}\right\}-2\right)} .
$$

Here we have used the commutation of $\check{p}_{1(2)}$ with $\check{G}_{1(2)}$ and $\check{G}_{c}$, which is a consequence of the normalization $\check{G}_{i}^{2}=\check{G}_{c}^{2}=1$, and the matrix property

$$
\check{A}^{2}=1 \Rightarrow[\check{A},\{\check{A}, \check{B}\}]=0 .
$$

We can solve Eq. (2) while assuming that $\check{p}_{1}$ and $\check{p}_{2}$ depend only on the anticommutator of the Green's functions of the leads, $\check{p}_{i}=\check{p}_{i}\left(\left\{\check{G}_{1}, \check{G}_{2}\right\}\right)$ (see the Appendix), and commute with $\check{G}_{1}, \check{G}_{2}$, and $\check{G}_{c}$ in accordance with Eq. (4). As a result, the Green's function of the cavity can be expressed in terms of Green's functions of the leads in the form

$$
\check{G}_{c}=\frac{\check{p}_{1}}{\check{c}} \check{G}_{1}+\frac{\check{p}_{2}}{\check{c}} \check{G}_{2},
$$

where the matrix $\check{c}=\check{c}\left(\left\{\check{G}_{1}, \check{G}_{2}\right\}\right)$ accounts for the normalization of $\check{G}_{c}$. From Eq. (5) and by using the normalization conditions $\check{G}_{c}^{2}=\check{G}_{1}^{2}=\check{G}_{2}^{2}=1$ we obtain the system of equations

$$
\begin{gathered}
\check{c}^{2}=\check{p}_{1}^{2}+\check{p}_{2}^{2}+\check{p}_{1} \check{p}_{2} \check{\mathcal{G}}, \\
\check{c} \check{\mathcal{G}}_{1}=2 \check{p}_{1}+\check{p}_{2} \check{\mathcal{G}}, \\
\check{c} \check{\mathcal{G}}_{2}=2 \check{p}_{2}+\check{p}_{1} \check{\mathcal{G}}^{\prime},
\end{gathered}
$$

where $\check{\mathcal{G}}_{i}=\left\{\check{G}_{i}, \check{G}_{c}\right\}$ and $\check{\mathcal{G}}=\left\{\check{G}_{1}, \check{G}_{2}\right\}$. This system can be treated, effectively, as a system of scalar equations because all matrices that appear in Eqs. (6)-(8) depend only on $\breve{\mathcal{G}}$ and commute with each other. 
The cumulant generating function $S(\chi)$ of charge transfer can be obtained as a sum of the actions of the connected pairs of nodes: ${ }^{44}$

$$
S(\chi)=-\frac{t_{0}}{2 h} \sum_{i=1,2} \sum_{n} \int d \mathcal{E} \operatorname{Tr} \ln \left(1+\frac{T_{n}^{(i)}}{4}\left(\check{\mathcal{G}}_{i}-2\right)\right),
$$

where the total measurement time $t_{0}$ is much larger than the characteristic time scale on which the current fluctuations are correlated. The $\chi$-independent term in the cumulant generating function [given by the normalization requirement $S(\chi$ $=0)=0$ ] is omitted for brevity throughout this article. Also, we recall that because of current conservation, it is sufficient to introduce a counting field $\chi$ at one lead only, while the full counting field dependence can be obtained by setting $\chi$ $=\chi_{1}-\chi_{2}$.

The cumulant generating function $S$ depends only on the anticommutator $\check{\mathcal{G}}(\chi)$ and is invariant to the exchange of the leads $\check{G}_{1} \leftrightarrow \check{G}_{2}$ or, equivalently, to exchange of the junctions $\left\{T_{n}^{(1)}\right\} \leftrightarrow\left\{T_{n}^{(2)}\right\}$ [see Eqs. (2) and (3)]. Therefore, the same invariance persists ${ }^{32,38,50}$ in all coherent (or low bias) transport properties of two-terminal double junctions-such as current (conductance), noise (Fano factor), and higher cumulants-independently of the type of leads or specific properties of the junctions. This invariance does not hold in the presence of dephasing, ${ }^{48,50}$ which can be modeled by an additional lead that carries the coherence leakage current. ${ }^{15-17,43}$

In the following we consider an analytically tractable case of a chaotic cavity coupled to the leads by two quantum point contacts with $N_{1}$ and $N_{2}$ open channels, respectively. The transmission eigenvalues of the contacts are $T_{n}^{(i)}=1$ for $n=1, \ldots, N_{i}$ and $T_{n}^{(i)}=0$ otherwise. From Eq. (3) and Eqs. (6)-(8) we find

$$
\check{c}=\frac{N_{1}+N_{2}}{2}\left(1+\sqrt{1-\frac{4 N_{1} N_{2}}{\left(N_{1}+N_{2}\right)^{2}} \frac{\check{\mathcal{G}}-2}{\check{\mathcal{G}}+2}}\right)^{-1},
$$

and

$$
\frac{\check{p}_{1,2}}{\check{c}}=\frac{1}{2}\left( \pm \frac{N_{1}-N_{2}}{N_{1}+N_{2}}+\sqrt{1-\frac{4 N_{1} N_{2}}{\left(N_{1}+N_{2}\right)^{2}} \frac{\check{\mathcal{G}}-2}{\check{\mathcal{G}}+2}}\right) .
$$

The Green's function of the cavity and cumulant generating function are given by Eqs. (5) and (9), respectively. A formally similar result has been obtained recently by Bulashenko ${ }^{38}$ using $2 \times 2$ Green's functions in Keldysh space which can be expanded over the Pauli matrices. Physically, this implies that the whole circuit is in the normal state, although it is permitted that the leads are nodes of a larger (normal-state) mesoscopic network. In our approach we do not rely on this expansion and make no assumptions on the particular matrix structure, except for the usual normalization condition. Therefore, Eqs. (10) and (11) are valid for any type of leads. For example, one lead can be superconducting, with the Green's function having Keldysh-Nambu matrix structure, or the chaotic cavity can be a part of the larger multiterminal network which consists of different hetero- junctions. Additional degrees of freedom-for instance, spin — can be included as well. We emphasize, again, that our solution only resorts to the normalization condition of the leads. In the case in which the cavity is part of a larger network, Green's functions $\check{G}_{1,2}$ have to be determined by circuit rules. The result for $\check{G}_{c}$ is valid in this case as well, which can simplify the numerical solution of larger circuits.

It is interesting to check that an alternative approach can give the same result. Coherent connectors in the circuit theory are described by a cumulant generating function of the form

$$
S(\chi)=-\frac{t_{0}}{2 h} \int d \mathcal{E} \int_{0}^{1} d \mathcal{T} \rho(\mathcal{T}) \operatorname{Tr} \ln \left(1+\frac{\mathcal{T}}{4}[\check{\mathcal{G}}(\chi)-2]\right),
$$

where $\rho(\mathcal{T})$ is the distribution of transmission eigenvalues $\left\{T_{n}\right\}$ for the composite junction. Using the distribution of transmission eigenvalues for an open chaotic cavity, ${ }^{16,42}$

$$
\rho_{c}(\mathcal{T})=\frac{\sqrt{N_{1} N_{2}}}{\pi} \frac{1}{\mathcal{T}} \sqrt{\frac{\mathcal{T}-\mathcal{T}_{0}}{(1-\mathcal{T})\left(1-\mathcal{T}_{0}\right)}},
$$

for $\mathcal{T}_{0}<\mathcal{T}<1$ and $\rho_{c}(\mathcal{T})=0$ otherwise, with $\mathcal{T}_{0}=\left(N_{1}\right.$ $\left.-N_{2}\right)^{2} /\left(N_{1}+N_{2}\right)^{2}$, we obtain $S(\chi)$ as given by Eqs. (9)-(11). This demonstrates the consistency of the circuit-theory approach with the random matrix theory of scattering matrices.

\section{SUPERCONDUCTOR-CHAOTIC- CAVITY-NORMAL-METAL JUNCTION}

In the following we calculate the statistics of charge transport through a chaotic cavity sandwiched between a superconductor and a normal-metal lead. We present a detailed analysis of the first three cumulants-current, current noise power, and the third cumulant of the current-for an open chaotic cavity at temperatures and bias voltages well below the superconducting gap $\Delta$, when Andreev scattering is the dominant process of the charge transfer. At low energies and temperatures the $4 \times 4$ matrix Green's function of the superconductor is $\check{G}_{S} \equiv \check{G}_{1}=\overline{1} \hat{\sigma}_{1}$ in the $\operatorname{Keldysh}(\overline{ }) \otimes \operatorname{Nambu}\left({ }^{\wedge}\right)$ space. The Green's function $\check{G}_{N}=\check{G}_{N}(\mathcal{E}, \chi) \equiv \check{G}_{2}$ of the normal-metal lead incorporates the counting field according to

$$
\check{G}_{N}=e^{-i(\chi / 2) \check{\tau}_{K}} \check{G}_{N}^{0} e^{i(\chi / 2) \check{\tau}_{K}},
$$

where $\check{\tau}_{K}=\bar{\tau}_{1} \hat{\sigma}_{3}$ and $\bar{\tau}_{i}$ and $\hat{\sigma}_{i}$ are Pauli matrices. The bare Green's function of the normal-metal lead is given by

$$
\check{G}_{N}^{0}=\left(\begin{array}{cc}
\hat{\sigma}_{3} & 2 \hat{K} \\
0 & -\hat{\sigma}_{3}
\end{array}\right), \quad \hat{K}=\left(\begin{array}{cc}
1-2 f_{N}^{+} & 0 \\
0 & 1-2 f_{N}^{-}
\end{array}\right),
$$

where $f_{N}^{ \pm}=\left\{\exp \left[( \pm \mathcal{E}+e V) / k_{B} T\right]+1\right\}^{-1}$ accounts for the voltage bias of the normal-metal lead, with the energy $\mathcal{E}$ measured in respect to the chemical potential of the superconductor. From Eqs. (9)-(11), we find the following expression for the cumulant generating function 


$$
S(\chi)=-\frac{t_{0}}{h} \int d \mathcal{E} \sum_{j=1,2} N_{j} \ln \left[r_{j}+\sqrt{r_{j}^{2}-64 N_{j}^{4}(1+a)}\right],
$$

where

$$
\begin{aligned}
r_{1(2)}= & a\left(N_{1}-N_{2}\right)^{2}+\left(3 N_{1(2)}+N_{2(1)}\right)^{2} \\
& +\sqrt{(1+a)\left[a\left(N_{1}-N_{2}\right)^{4}+\left(N_{1}^{2}+N_{2}^{2}+6 N_{1} N_{2}\right)^{2}\right]} .
\end{aligned}
$$

Here, $a=\left(e^{2 i \chi}-1\right) \tilde{f}_{N}^{+} \tilde{f}_{N}^{-}+\left(e^{-2 i \chi}-1\right) f_{N}^{+} f_{N}^{-}$and $\tilde{f}_{N}^{ \pm}=1-f_{N}^{ \pm}$, with $a$ being related to the double-degenerate eigenvalues of $\check{\mathcal{G}}$ $=\left\{\check{G}_{S}, \check{G}_{N}\right\}$ given by $\lambda_{1,2}= \pm 2 i \sqrt{a}$. From Eq. (16) we obtain the average current, the current noise power, and the third cumulant according to $I=\left.i\left(e / t_{0}\right) \partial_{\chi} S\right|_{\chi=0}, \quad P_{I}$ $=\left.\left(2 e^{2} / t_{0}\right) \partial_{\chi}^{2} S\right|_{\chi=0}$, and $C_{I}=-\left.i\left(e^{3} / t_{0}\right) \partial_{\chi}^{3} S\right|_{\chi=0}$, respectively. They are

$$
\begin{gathered}
I=\frac{G_{S}}{2 e} \int d \mathcal{E}\left(\tilde{f}_{N}^{+} \tilde{f}_{N}^{-}-f_{N}^{+} f_{N}^{-}\right), \\
P_{I}=2 G_{S} \int d \mathcal{E}\left[\left(\tilde{f}_{N}^{+} \tilde{f}_{N}^{-}+f_{N}^{+} f_{N}^{-}\right)-\gamma_{1}\left(\tilde{f}_{N}^{+} \tilde{f}_{N}^{-}-f_{N}^{+} f_{N}^{-}\right)^{2}\right],
\end{gathered}
$$

and

$$
\begin{aligned}
C_{I}= & 2 e G_{S} \int d \mathcal{E}\left(\tilde{f}_{N}^{+} \tilde{f}_{N}^{-}-f_{N}^{+} f_{N}^{-}\right)\left[1-3 \gamma_{1}\left(\tilde{f}_{N}^{+} \tilde{f}_{N}^{-}+f_{N}^{+} f_{N}^{-}\right)\right. \\
& \left.+2 \gamma_{2}\left(\tilde{f}_{N}^{+} \tilde{f}_{N}^{-}-f_{N}^{+} f_{N}^{-}\right)^{2}\right],
\end{aligned}
$$

with the total conductance of S-cavity-N junction,

$$
G_{S}=\frac{2 e^{2}}{h}\left(N_{1}+N_{2}\right)\left(1-\frac{N_{1}+N_{2}}{q}\right),
$$

and the low-temperature Fano factor ${ }^{15}$

$$
F_{S}=\frac{16 N_{1}^{2} N_{2}^{2}\left(N_{1}+N_{2}\right)}{q^{4}\left[q-\left(N_{1}+N_{2}\right)\right]} .
$$

Here $q=\sqrt{\left(N_{1}+N_{2}\right)^{2}+4 N_{1} N_{2}}, \quad \gamma_{1}=1-F_{S} / 2$, and $\gamma_{2}=1-F_{S}$ $\left[1-2 N_{1} N_{2}\left(2 N_{1}+N_{2}\right)\left(N_{1}+2 N_{2}\right) / q^{4}\right]$. After the energy integration in Eq. (18), the usual relation $I=G_{S} V$ is obtained, while the integration in Eqs. (19) and (20) yields

$$
\begin{aligned}
\frac{P_{I}}{4 G_{S} k_{B} T} & =1+\left(F_{S} / 2\right)[v \operatorname{coth}(v)-1] \\
& = \begin{cases}1+\left(F_{S} / 6\right) v^{2}, & |v| \ll 1, \\
1-F_{S} / 2+\left(F_{S} / 2\right)|v|, & |v| \gg 1,\end{cases}
\end{aligned}
$$

and

$$
\frac{C_{I}}{e G_{S} k_{B} T}=12\left(\gamma_{1}-\gamma_{2}\right)\left[\operatorname{coth}(v)+v\left(\frac{1-\gamma_{2}}{3\left(\gamma_{1}-\gamma_{2}\right)}-\operatorname{coth}^{2}(v)\right)\right]
$$

$$
= \begin{cases}2 F_{S} v, & |v| \ll 1, \\ \pm 12\left(\gamma_{1}-\gamma_{2}\right)+4\left(1-3 \gamma_{1}+2 \gamma_{2}\right) v, & \pm v \gg 1,\end{cases}
$$

respectively, with $v=e V / k_{B} T$.

The cumulant generating function, Eq. (16), takes into account the superconductor proximity effect in the quasiclassical approximation as well as interchannel mixing inside the cavity in the presence of quantum point contacts. In comparison with the normal-state junction (see Ref. 38), Eqs. (18)-(20) contain products of electron and hole distribution functions due to the Andreev process, which is the mechanism of the charge transport. For example, the term $f_{N}^{+} f_{N}^{-}$ $=f_{N}^{+}\left(1-\tilde{f}_{N}^{-}\right)$can be interpreted as the probability for an electron emerging from the lead $\mathrm{N}$ to be reflected back as a hole, where $f_{N}^{+}$and $\tilde{f}_{N}^{-}$are the electron- and hole-state occupation numbers. The energy-independent prefactors $G_{S}, \gamma_{1}$, and $\gamma_{2}$ are also modified by the electron-hole correlations introduced by the superconductor. This change of transport properties due to superconductor proximity effect can be revealed by considering a general $\mathrm{S} / \mathrm{N}$ junction with transmission distribution $\rho(\mathcal{T})$, at low temperatures and bias voltages $\left(k_{B} T,|e V| \ll \Delta\right)$. In this case Eq. (12) reduces to

$$
S(\chi)=-\frac{t_{0}}{2 h} \int d \mathcal{E} \int_{0}^{1} d R_{A} \rho_{A}\left(R_{A}\right) \ln \left[1+R_{A} a(\chi)\right]
$$

where $\lambda_{1,2}= \pm 2 i \sqrt{a}$ are double-degenerate eigenvalues of $\check{\mathcal{G}}$. In the fully coherent regime, which we consider here, the distribution of Andreev reflection eigenvalues $\rho_{A}\left(R_{A}\right)$ is simply related to the distribution of transmissions $\rho(\mathcal{T})$ of the corresponding normal-state junction: $\rho_{A}\left(R_{A}\right)=2 \rho(\mathcal{T}) d \mathcal{T} / d R_{A}$. The probability of the Andreev reflection is given by $R_{A}$ $=\mathcal{T}^{2} /(2-\mathcal{T})^{2}$ and appears in Eq. (27) due to electron-hole symmetry at energies well below the superconducting gap and the inverse dwell time in the cavity. Normal scattering processes are suppressed, and $S(\chi)$ depends on the counting field through $e^{ \pm 2 i \chi}$, which accounts for the effective charge of $2 e$ that is transferred across the structure in each elementary event of Andreev scattering. In the case of strong electronhole dephasing within the structure, electrons and holes decouple and the system can be mapped onto an effective normal-state junction, ${ }^{45,50}$ for which the cumulant generating function is given by Eq. (12) with the corresponding modification of transmission distribution $\rho(\mathcal{T})$ and boundary conditions $\check{\mathcal{G}}$. In the crossover regime, transport through the structure is not simply related to the normal-state transmission properties and can be described by an effective energydependent distribution ${ }^{47} \rho_{A}$ which takes into account the effects of dephasing (coherence leakage currents) at characteristic energies on the order of Thouless energy.

Expanding Eq. (27) in the $\chi$ field and taking the derivatives, we obtain the current, current noise power, and the third cumulant in the coherent regime as given by Eqs. (18)-(20), with conductance 

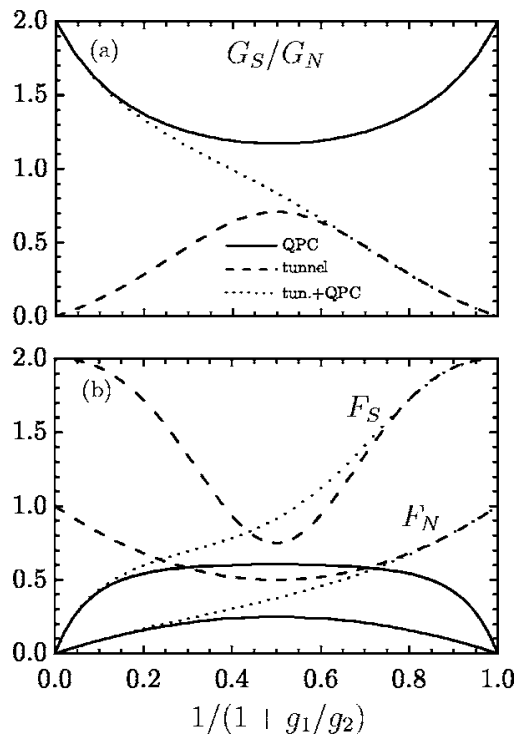

FIG. 2. Conductance $G_{S}$ [panel (a)] and the Fano factor $F_{S}$ [panel (b)] of the S-cavity-N junction as a function of the junction asymmetry $1 /\left(1+g_{1} / g_{2}\right)$. Results for three different couplings of a cavity to the leads are shown for comparison: coupling by quantum point contacts (solid curves), tunnel junctions (dashed curves), and coupling by tunnel junction from the $\mathrm{S}$ side and quantum point contact from the $\mathrm{N}$ side (dotted curves). Conductance is normalized to the normal-state value $G_{N}=g_{1} g_{2} /\left(g_{1}+g_{2}\right)$, with $g_{i}=\left(2 e^{2} / h\right) N_{i}$ for quantum point contacts and $g_{i}=\left(2 e^{2} / h\right) \Sigma_{n} T_{n}^{(i)}$ for tunnel junctions. Fano factors $F_{N}$ for the corresponding normal-state junctions are shown in panel (b) for comparison.

$$
\widetilde{G}_{S}=2 \int_{0}^{1} d \mathcal{T} \rho(\mathcal{T}) R_{A}
$$

$\gamma_{1}=2 \widetilde{G}_{S}^{-1} \int_{0}^{1} d \mathcal{T} \rho(\mathcal{T}) R_{A}^{2}$, and $\gamma_{2}=2 \widetilde{G}_{S}^{-1} \int_{0}^{1} d \mathcal{T} \rho(\mathcal{T}) R_{A}^{3}$, where $\widetilde{G}_{S}$ $=G_{S} /\left(2 e^{2} / h\right)$. In particular, the Fano factor and the slope of the third cumulant at high bias are given by

$$
F_{S}=\frac{4}{\widetilde{G}_{S}} \int_{0}^{1} d \mathcal{T} \rho(\mathcal{T}) R_{A}\left(1-R_{A}\right)
$$

and

$$
\frac{\partial C_{I}}{\partial\left(e^{2} I\right)}=\frac{8}{\widetilde{G}_{S}} \int_{0}^{1} d \mathcal{T} \rho(\mathcal{T}) R_{A}\left(1-R_{A}\right)\left(1-2 R_{A}\right),
$$

respectively. These expressions are similar to the normalstate ones except for the effective charge doubling and the presence of the Andreev reflection probability $R_{A}$ instead of normal transmission $\mathcal{T}$, in agreement with previous results obtained within the scattering approach. ${ }^{6}$ Using the transmission distribution $\rho_{c}(\mathcal{T})$ given by Eq. (13), we recover the results for an open asymmetric cavity, which were obtained from the circuit theory without the explicit knowledge of $\rho(\mathcal{T})$ for the composite junction.

The total conductance $G_{S}$ [normalized to the normal-state value $\left.G_{N}=g_{1} g_{2} /\left(g_{1}+g_{2}\right)\right]$ and the Fano factor $F_{S}$ of the S-cavity-N junction are shown in Fig. 2 as functions of the junction asymmetry and for different couplings between the cavity and leads. For the symmetric quantum-point-contact coupling $g_{1} / g_{2}=1$, the conductance ratio has the minimal value $G_{S} / G_{N}=2(2-\sqrt{2}) \approx 1.17$, while the Fano factor is maximal, $F_{S}=(\sqrt{2}+1) / 4 \approx 0.60$. In the highly asymmetric limit, $G_{S} / G_{N}=2$ and $F_{S}=8 g_{\min } / g_{\max } \approx 0$ (Fig. 2, solid curves). The vanishing of the shot noise in this case is due to the perfect transparency of the dominant (the one which is weakly coupled) quantum point contact. For the case of two tunnel junctions ${ }^{32,48}$ instead of quantum point contacts, the trend is opposite: for the symmetric coupling, $G_{S} / G_{N}$ $=1 / \sqrt{2} \approx 0.71$ is maximal and $F_{S}=3 / 4$ is minimal, and for the highly asymmetric coupling, $G_{S} / G_{N}=g_{\min } / g_{\max } \approx 0$ and $F_{S}=2$ (see Fig. 2, dashed curves). We point out that these different trends can be used to probe the quality of the contacts. The dotted curves in Fig. 2 show numerical results for the conductance and the Fano factor of a cavity coupled to a superconductor by a tunnel junction and to a normal lead by quantum point contact. We find that the transport properties of the system are not affected by the type of coupling to the superconductor when the quantum point contact on the normal side dominates. This limit is reached at the conductance ratio $g_{1} / g_{2} \gtrsim 5$ of the tunnel- and quantum-point-contact coupling of the cavity to the leads. Therefore, the junction that corresponds to the model of an open chaotic cavity can be realized either with two good quality quantum point contacts from the both sides or it can be an asymmetric junction with only the normal-side quantum point contact of a high quality. The latter is easier to fabricate, and the required asymmetry can be achieved by increasing the contact area of the cavity to the superconductor. Experimentally, the conductance and current noise power have been measured recently by Choi et $a l .,{ }^{25}$ in a setup which is very similar to the system we have analyzed. As the estimates from Ref. 25 show, it is possible to fabricate a high-quality contact between cavity and superconductor. The measured values of the Fano factors $F_{S}$ $=0.58 \pm 0.10$ and $F_{N}=0.25 \pm 0.04$ across the junction in the superconducting- and normal-state regimes, respectively, are in agreement with the model of a symmetric open chaotic cavity [compare with solid curves in Fig. 2(b)]. However, the measured conductance ratio $G_{S} / G_{N} \approx 0.90$ is in discrepancy with the conductance ratio $G_{S} / G_{N}=1.17$ predicted by this simple model. The difference may originate from the inelastic quasiparticle scattering at the disordered superconductor interface, nonuniversal correction due to relatively large openings of the cavity, ${ }^{30}$ or dephasing of quasiparticles due to an additional lead which is left floating in the experiment.

At high temperatures $k_{B} T \gg|e V|$, current noise power is thermally dominated and linear in conductance and temperature [see Eq. (24)], as expected from the fluctuationdissipation theorem. Thus, to extract the Fano factor from the current noise power measurement, it is necessary to be in the low-temperature, shot-noise regime $k_{B} T \ll|e V|$. Experimentally, this requires high bias voltages at which nonlinear $I-V$ characteristics occur, especially in a strongly interacting electron systems with a difficulty how to distinguish the shot noise contribution from the contribution of thermal noise modified by nonlinear conductance.

Finally, we point out the difference between the superconducting and normal-state asymptotic behavior of the third cumulant at high biases. For an asymmetric open cavity 

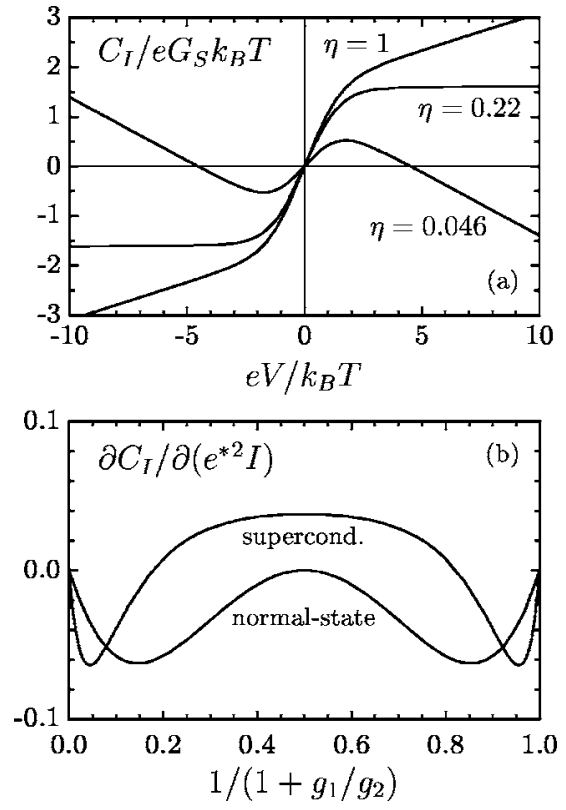

FIG. 3. The third cumulant as a function of bias-to-temperature ratio [panel (a)], shown for three characteristic junction asymmetries: $C_{I}$ has the maximal positive slope at high biases for symmetric coupling $\eta=g_{\min } / g_{\max }=1$, saturates at high biases for $\eta \approx 0.22$, and has maximal negative slope for $\eta \approx 0.046$. The high-bias slope of the third cumulant is shown in panel (b) as a function of the junction asymmetry, with the normal-state value given for comparison. Effective charge is $e^{*}=2 e$ and $e^{*}=e$ for the superconducting and normal-state junction, respectively.

coupled to normal-metal leads, the slope of the voltagedependent third cumulant is negative, reaching zero for the symmetric cavity. ${ }^{38}$ When one lead is superconducting, this slope is negative for highly asymmetric couplings and positive for symmetric couplings [Fig. 3(b)], with the crossover at $\eta=g_{\text {min }} / g_{\text {max }}=\sqrt{3+2 \sqrt{2}}-\sqrt{2+2 \sqrt{2}} \approx 0.22$. Thus, in the normal-state regime the third cumulant changes sign at high enough biases, while in the superconducting case this happens only if the junction is sufficiently asymmetric. This difference originates from the interchannel mixing inside the cavity in the presence of the superconducting proximity effect $^{32}$ and can be attributed to the skewness ${ }^{38}$ of the Andreev reflection probability distribution function $\rho_{A}\left(R_{A}\right)$ $=2 \rho_{c}(\mathcal{T}) d \mathcal{T} / d R_{A} \quad$ [compare with Eqs. (28)-(30)]. For the normal-state symmetric cavity, the transmission distribution is symmetric-i.e., $\rho_{c}(\mathcal{T})=\rho_{c}(1-\mathcal{T})$ (Fig. 4)-leading to the saturation of the third cumulant at high bias. If the junction is asymmetric, then the gap at low transmissions opens at $0<\mathcal{T}<\mathcal{T}_{0}$, shifting the weight of the distribution towards the open channels, $\rho_{c}(\mathcal{T})<\rho_{c}(1-\mathcal{T})$ for $0<\mathcal{T}<1 / 2$, and the high-bias slope of the third cumulant becomes negative. When one lead is superconducting, the weight of distribution $\rho_{A}\left(R_{A}\right)$ for the symmetric cavity is at low values of the Andreev reflection probabilities, $\rho_{A}\left(R_{A}\right)>\rho_{A}\left(1-R_{A}\right)$ for $0<R_{A}$ $<1 / 2$ (Fig. 4), leading to the positive high-bias slope of the third cumulant. Only for large asymmetries of the junction does the gap that opens at low $R_{A}$ prevail, and the distribution $\rho_{A}$ shifts towards the open Andreev channels and the third cumulant becomes negative (Fig. 3). It is interesting to

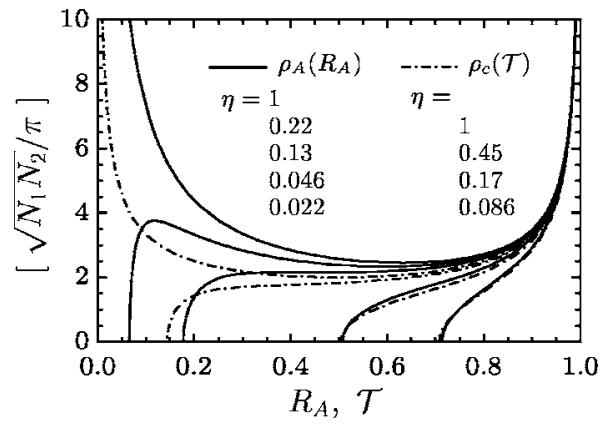

FIG. 4. Distribution of the Andreev reflection probabilities $\rho_{A}\left(R_{A}\right)=2 \rho_{c}(\mathcal{T}) d \mathcal{T} / d R_{A}$ (solid curves) and the distribution of transmission eigenvalues $\rho_{c}(\mathcal{T})$ (dash-dotted curves) for an open chaotic cavity, shown for different asymmetries of the junction $\eta$ $=g_{\min } / g_{\max }$ (from top to bottom).

note that the maximally negative slopes of the third cumulant in the normal and in the Andreev case are approximately equal (if the latter are corrected for the effective charge). From Fig. 4 it is seen that the eigenvalue distributions are very similar for these values, with the effect of a superconductor being the change of a junction asymmetry. We believe it would be interesting to confirm these predictions experimentally. They provide much more detailed information on the transmission eigenvalue and Andreev reflection eigenvalue distributions, which go beyond the information obtained from conductance and noise measurements.

\section{CONCLUSION}

We have studied the charge transport statistics in coherent two-terminal double junctions within the circuit theory of mesoscopic transport. We have shown that the system of circuit-theory matrix equations for the Green's function of the central cavity can be solved, effectively, as a system of scalar equations independently on the type of the leads. For an asymmetric cavity coupled to the leads by quantum point contacts, the Green's function is expressed in a closed analytical form in terms of the matrix Green's functions of the leads. The full counting statistics and the first three cumulants are obtained for a special case of an open cavity between a superconductor and a normal metal, at temperatures and bias voltages below the superconducting gap.

The same results can be obtained by applying the circuit theory while considering the whole structure as a single connector, with the cumulant generating function integrated over the distribution of transmission eigenvalues of the composite junction. This approach manifestly reveals how the subgap transport in $\mathrm{S} / \mathrm{N}$ structures is affected both by the effective charge doubling due to the Andreev process and by modification of the transmission properties due to electron-hole correlations introduced by the superconductor.

For an open cavity, the Fano factor is enhanced with respect to the corresponding normal-state junction, in agreement with the recent experimental results by Choi et al. ${ }^{25}$ where the high-quality contacts between a cavity and superconductor have been made. In comparison to the tunnel coupling, the conductance and Fano factor exhibit opposite 
trends as a function of the junction asymmetry, which can be used experimentally to probe the quality of the contacts. The third cumulant is strongly affected by the presence of a superconductor. In contrast to the normal-state case, in which the third cumulant changes the sign at high enough biases, in the case in which one lead is superconducting this happens only if the junction is sufficiently asymmetric. This difference originates from the skewness ${ }^{38}$ of the Andreev reflection distribution function, which is in favor of closed Andreev channels for a moderate asymmetries of the junction.

\section{ACKNOWLEDGMENTS}

We are grateful to Christoph Bruder, Peter Samuelsson, and Oleg Bulashenko for useful discussions. This work has been supported by the Swiss NSF and the NCCR "Nanoscience."

\section{APPENDIX}

Here we show that $\check{p}_{i}$ given by Eq. (3) depends only on the anticommutator of the Green's functions of the leads, $\check{p}_{i}=\check{p}_{i}\left(\left\{\check{G}_{1}, \check{G}_{2}\right\}\right)$, under the assumption that it is possible to expand $\check{G}_{c}$ in a series over the products of $\check{G}$ matrices. In this case $\check{p}_{i}$ is also a series over $\check{G}$ matrices, which we denote as $\check{p}_{i}=\check{p}_{i}\left(\check{G}_{1}, \check{G}_{2}\right)$. In the following we consider only $\check{p}_{1}$, while the reasoning for $\check{p}_{2}$ is analogous. In $\check{p}_{1}\left(\check{G}_{1}, \check{G}_{2}\right)$ we separate the term $\check{\varphi}\left(\check{G}_{1} \check{G}_{2}, \check{G}_{2} \check{G}_{1}\right)$ which contains a sum of products with an even number of $\check{G}$ matrices, and the term with the sum of odd-number products. The latter is of the form
$\check{G}_{1} \check{\phi}\left(\check{G}_{1} \check{G}_{2}, \check{G}_{2} \check{G}_{1}\right)$, where we used $\check{G}_{1}^{2}=\check{G}_{2}^{2}=1$. As a result, $\check{p}_{1}\left(\check{G}_{1}, \check{G}_{2}\right)=\check{\varphi}\left(\check{G}_{1} \check{G}_{2}, \check{G}_{2} \check{G}_{1}\right)+\check{G}_{1} \check{\phi}\left(\check{G}_{1} \check{G}_{2}, \check{G}_{2} \check{G}_{1}\right)$. Now we investigate the structure of $\check{\varphi}$ and $\check{\phi}$. First we express $\check{G}_{1} \check{G}_{2}$ and $\check{G}_{2} \check{G}_{1}$ in terms of $\left\{\check{G}_{1}, \check{G}_{2}\right\}$ and $\left[\check{G}_{1}, \check{G}_{2}\right]$, and then expand $\check{\varphi}$ $=\check{\varphi}\left(\left\{\check{G}_{1}, \check{G}_{2}\right\},\left[\check{G}_{1}, \check{G}_{2}\right]\right)$ in a series of $\left[\check{G}_{1}, \check{G}_{2}\right]$. Even powers of $\left[\check{G}_{1}, \check{G}_{2}\right]$ can be expressed in terms of $\left\{\check{G}_{1}, \check{G}_{2}\right\}$ by using the identity $\left[\check{G}_{1}, \check{G}_{2}\right]^{2}=\left\{\check{G}_{1}, \check{G}_{2}\right\}^{2}-4$. Thus, $\check{\varphi}$ is of the form $\check{\varphi}=\check{\alpha}+\check{\beta}\left[\check{G}_{1}, \check{G}_{2}\right]$, where $\check{\alpha}=\check{\alpha}\left(\left\{\check{G}_{1}, \check{G}_{2}\right\}\right)$ and $\check{\beta}=\check{\beta}\left(\left\{\check{G}_{1}, \check{G}_{2}\right\}\right)$ depend only on the anticommutator [and hence they commute with $\check{G}_{1}, \check{G}_{2}$, and $\left.\check{G}_{c}=\check{G}_{c}\left(\check{G}_{1}, \check{G}_{2}\right)\right]$. The same is true for $\check{\phi}$-i.e., $\check{\phi}=\check{\alpha}^{\prime}+\check{\beta}^{\prime}\left[\check{G}_{1}, \check{G}_{2}\right]$, where $\check{\alpha}^{\prime}=\check{\alpha}^{\prime}\left(\left\{\check{G}_{1}, \check{G}_{2}\right\}\right)$ and $\check{\beta}^{\prime}$ $=\check{\beta}^{\prime}\left(\left\{\check{G}_{1}, \check{G}_{2}\right\}\right)$. Therefore,

$$
\check{p}_{1}=\check{\alpha}+\check{\beta}\left[\check{G}_{1}, \check{G}_{2}\right]+\check{\alpha}^{\prime} \check{G}_{1}+\check{\beta}^{\prime} \check{G}_{1}\left[\check{G}_{1}, \check{G}_{2}\right] .
$$

On the other hand, $\left[\check{G}_{1}, \check{p}_{1}\right]=0$ in accordance with Eqs. (3) and (4), and we have

$$
\left[\check{G}_{1}, \check{p}_{1}\right]=2 \check{\beta} \check{G}_{1}\left[\check{G}_{1}, \check{G}_{2}\right]+2 \check{\beta}^{\prime}\left[\check{G}_{1}, \check{G}_{2}\right]=0 .
$$

From Eqs. (A1) and (A2) we find that $\check{p}_{1}$ has the following structure: $\check{p}_{1}=\check{\alpha}+\check{\alpha}^{\prime} \check{G}_{1}$. After substituting the expression $\check{p}_{1} \check{G}_{1}=\check{\alpha} \check{G}_{1}+\check{\alpha}^{\prime}$ back into Eq. (2) from which $\check{G}_{c}$ is to be obtained, the term $\check{\alpha}^{\prime}$ vanishes because it commutes with $\check{G}_{c}$. Thus, when solving for $\check{G}_{c}$ we can assume that $\check{p}_{1}$ $=\check{\alpha}\left(\left\{\check{G}_{1}, \check{G}_{2}\right\}\right)$ without loss of generality. A similar consideration holds for $\check{p}_{2}$.
${ }^{1}$ G. E. Blonder, M. Tinkham, and T. M. Klapwijk, Phys. Rev. B 25, 4515 (1982).

${ }^{2}$ A. F. Volkov, A. V. Zaitsev, and T. M. Klapwijk, Physica C 210, 21 (1993).

${ }^{3}$ V. A. Khlus, Zh. Eksp. Teor. Fiz. 93, 2179 (1987) [Sov. Phys. JETP 66, 1243 (1987)].

${ }^{4}$ Y. Takane and H. Ebisawa, J. Phys. Soc. Jpn. 61, 2858 (1992).

${ }^{5}$ C. W. J. Beenakker, Phys. Rev. B 46, R12841 (1992).

${ }^{6}$ M. J. M. de Jong and C. W. J. Beenakker, Phys. Rev. B 49, 16070 (1994).

${ }^{7}$ T. Martin, Phys. Lett. A 220, 137 (1996).

${ }^{8}$ C. J. Lambert, J. Phys.: Condens. Matter 3, 6579 (1991).

${ }^{9}$ M. P. Anantram and S. Datta, Phys. Rev. B 53, 16390 (1996).

${ }^{10}$ A. L. Fauchère, G. B. Lesovik, and G. Blatter, Phys. Rev. B 58, 11177 (1998).

${ }^{11}$ M. Schechter, Y. Imry, and Y. Levinson, Phys. Rev. B 64, 224513 (2001)

${ }^{12}$ K. E. Nagaev and M. Büttiker, Phys. Rev. B 63, 081301(R) (2001).

${ }^{13}$ F. Pistolesi, G. Bignon, and F. W. J. Hekking, Phys. Rev. B 69, 214518 (2004).

${ }^{14}$ M. Büttiker, Phys. Rev. B 46, 12485 (1992).

${ }^{15}$ M. J. M. de Jong and C. W. J. Beenakker, in Mesoscopic Electron Transport, edited by L. P. Kouwenhoven, G. Schön, and L. L.
Sohn (Kluwer, Dordrecht, 1997).

${ }^{16}$ C. W. J. Beenakker, Rev. Mod. Phys. 69, 731 (1997).

${ }^{17}$ Ya. M. Blanter and M. Büttiker, Phys. Rep. 336, 1 (2000).

${ }^{18}$ Quantum Noise in Mesoscopic Physics, edited by Yu. V. Nazarov (Kluwer, Dordrecht, 2003).

${ }^{19}$ R. J. Schoelkopf, P. J. Burke, A. A. Kozhevnikov, D. E. Prober, and M. J. Rooks, Phys. Rev. Lett. 78, 3370 (1997).

${ }^{20}$ M. Henny, S. Oberholzer, C. Strunk, and C. Schönenberger, Phys. Rev. B 59, 2871 (1999).

${ }^{21}$ S. Oberholzer, E. V. Sukhorukov, C. Strunk, C. Schönenberger, T. Heinzel, and M. Holland, Phys. Rev. Lett. 86, 2114 (2001); S. Oberholzer, E. V. Sukhorukov, C. Strunk, and C. Schönenberger, Phys. Rev. B 66, 233304 (2002); S. Oberholzer, E. V. Sukhorukov, and C. Schönenberger, Nature (London) 415, 765 (2002).

${ }^{22}$ X. Jehl, M. Sanquer, R. Calemczuk, and D. Mailly, Nature (London) 405, 50 (2000); X. Jehl and M. Sanquer, Phys. Rev. B 63, 052511 (2001).

${ }^{23}$ A. A. Kozhevnikov, R. J. Schoelkopf, and D. E. Prober, Phys. Rev. Lett. 84, 3398 (2000).

${ }^{24}$ F. Lefloch, C. Hoffmann, M. Sanquer, and D. Quirion, Phys. Rev. Lett. 90, 067002 (2003).

${ }^{25}$ B.-R. Choi, A. E. Hansen, T. Kontos, C. Hoffmann, S. Oberholzer, W. Belzig, C. Schönenberger, T. Akazaki, and H. Takay- 
anagi, Phys. Rev. B 72, 024501 (2005).

${ }^{26}$ B. Reulet, J. Senzier, and D. E. Prober, Phys. Rev. Lett. 91, 196601 (2003).

${ }^{27}$ Yu. Bomze, G. Gershon, D. Shovkun, L. S. Levitov, and M. Reznikov, cond-mat/0504382 (unpublished).

${ }^{28}$ Yu. V. Nazarov, Phys. Rev. Lett. 73, 134 (1994).

${ }^{29}$ E. V. Sukhorukov and D. Loss, Phys. Rev. Lett. 80, 4959 (1998).

${ }^{30}$ Ya. M. Blanter and E. V. Sukhorukov, Phys. Rev. Lett. 84, 1280 (2000).

${ }^{31}$ A. F. Andreev, Zh. Eksp. Teor. Fiz. 46, 1823 (1964) [Sov. Phys. JETP 19, 1228 (1964)].

${ }^{32}$ W. Belzig, in Ref. 18, p. 463.

${ }^{33}$ B. A. Muzykantskii and D. E. Khmelnitskii, Phys. Rev. B 50, 3982 (1994).

${ }^{34}$ J. Torrès, T. Martin, and G. B. Lesovik, Phys. Rev. B 63, 134517 (2001).

${ }^{35}$ L. S. Levitov and G. B. Lesovik, JETP Lett. 58, 230 (1993); L. S. Levitov, H. W. Lee, and G. B. Lesovik, J. Math. Phys. 37, 4845 (1996); L. S. Levitov, in Ref. 18, p. 373.

${ }^{36}$ H. Lee, L. S. Levitov, and A. Yu. Yakovets, Phys. Rev. B 51, 4079 (1995).

${ }^{37}$ L. S. Levitov and M. Reznikov, Phys. Rev. B 70, 115305 (2004); cond-mat/0111057 (unpublished).

${ }^{38}$ O. M. Bulashenko, J. Stat. Mech.: Theory Exp., P08013 (2005).
${ }^{39}$ K. E. Nagaev, Phys. Rev. B 66, 075334 (2002); K. E. Nagaev, P. Samuelsson, and S. Pilgram, ibid. 66, 195318 (2002).

${ }^{40}$ Yu. V. Nazarov, Ann. Phys. 8, SI-193 (1999).

${ }^{41}$ W. Belzig and Yu. V. Nazarov, Phys. Rev. Lett. 87, 197006 (2001).

${ }^{42}$ Yu. V. Nazarov in Quantum Dynamics of Submicron Structures, edited by H. A. Cerdeira, B. Kramer, and G. Schön (Kluwer, Dordrecht, 1995), p. 687.

${ }^{43}$ Yu. V. Nazarov, Superlattices Microstruct. 25, 1221 (1999).

${ }^{44}$ Yu. V. Nazarov and D. A. Bagrets, Phys. Rev. Lett. 88, 196801 (2002).

${ }^{45}$ W. Belzig and P. Samuelsson, Europhys. Lett. 64, 253 (2003).

${ }^{46}$ W. Belzig and Yu. V. Nazarov, Phys. Rev. Lett. 87, 067006 (2001).

${ }^{47}$ P. Samuelsson, W. Belzig, and Yu. V. Nazarov, Phys. Rev. Lett. 92, 196807 (2004).

${ }^{48}$ P. Samuelsson, Phys. Rev. B 67, 054508 (2003).

${ }^{49}$ M. Houzet and F. Pistolesi, Phys. Rev. Lett. 92, 107004 (2004).

${ }^{50}$ G. Bignon, F. Pistolesi, and M. Houzet, cond-mat/0502453 (unpublished).

${ }^{51}$ P. Samuelsson and M. Büttiker, Phys. Rev. Lett. 89, 046601 (2002); Phys. Rev. B 66, 201306(R) (2002).

${ }^{52}$ J. Börlin, W. Belzig, and C. Bruder, Phys. Rev. Lett. 88, 197001 (2002). 